\title{
Trial Length
}

National Cancer Institute

\section{Source}

National Cancer Institute. Trial Length. NCI Thesaurus. Code C49697.

The anticipated length of a clinical trial measured as a unit of time. 